Article

\title{
The Relationship between the Angle of Curvature of the Spine and SEMG Amplitude of the Erector Spinae in Young School-Children
}

\author{
Jacek Wilczyński *(D), Przemysław Karolak, Sylwia Janecka, Magdalena Kabała and \\ Natalia Habik-Tatarowska \\ Department Posturology, Hearing and Balance Rehabilitation, Institute of Physiotherapy, \\ Faculty of Medicine and Health Sciences, Jan Kochanowski University in Kielce, Al. IX Wieków Kielc 19, \\ 25-317 Kielce, Poland \\ * Correspondence: jwilczynski@onet.pl
}

Received: 11 July 2019; Accepted: 29 July 2019; Published: 1 August 2019

check for updates

\begin{abstract}
The aim of the study was to analyze the relationship between the angle of spinal curvature and surface electromyography (SEMG) amplitude of the erector spinae in young school-children. A total of 251 children aged 7-8 participated in the study. The analysis involved 103 (41\%) children with scoliosis, $141(56.17 \%)$ with scoliotic posture, and seven (3.0\%) with normal posture. Body posture was evaluated using the Diers formetric III 4D optoelectronic method. Analysis of SEMG amplitude of the erector spinae was performed with the Noraxon TeleMyo DTS apparatus. A significant correlation was found between the angle of spinal curvature and the SEMG amplitude of the erector spinae. The most important and statistically significant predictor of the SEMG amplitude and scoliosis angle in the scoliosis group was the standing position, chest segment, right side. The largest generalized SEMG amplitude of the erector spinae occurred in both boys and girls with scoliosis. Impaired balance of muscle tension in the erector spinae can trigger a set of changes that create a clinical and anatomopathological image of spinal curvature.
\end{abstract}

Keywords: scolisis; electromyographic examination; Diers formetric III 4D

\section{Introduction}

The causes of idiopathic scoliosis have not been explained to date. There is no universally accepted theory of scoliosis etiopathogenesis. There is a consensus that idiopathic scoliosis is multifactorial. Extensive literature in this field describes abnormalities at the level of systems, organs, tissues, cells, particles, and genes, without prejudging their primary or secondary nature [1]. In an etiopathogenetic sense, scoliosis is only a symptom, an external expression of an unrecognized pathology that can occur in different places of the spine [2]. Although scoliosis is obviously a distortion of posture and body structure, it is also the effect of its compensatory abilities, allowing the preservation of the position of the head and shoulder girdle over the pelvis [3]. The final shape of the body is the result of deforming processes and compensatory reactions. It is justifiable to talk about etiological factors, and not about a specific theory, e.g., genetic or metabolic scoliosis [4].

Currently, the multi-factor concept in this genetically conditioned pathology of the central nervous system has the most supporters. This pathology applies, in particular, to the broadly understood postural system [5]. It affects, most likely, the system of deep back muscles (erector spinae), on the growing spine of a child with individually variable and multifactorial susceptibility to distortion [6]. Progression of scoliosis is associated with growth and biomechanical factors.

Among theories on the etiology of scoliosis, the concept of disturbances in the balance of muscle tension is of special importance. In maintaining the course of fibers of individual muscles in scoliosis, 
an important role is played by the thoracolumbar fascia, which presses the erector spinae muscle to the spine and prevents greater dislocations [7]. The uneven tension of the erector spinae visible on both sides and at different levels of the spine, visible in the surface electromyography (SEMG) amplitude record, is related to the mass movement of this muscle. The main mass of the erector spinae muscle lies in the groove restricted by spinal processes of the vertebrae on one side and the arch lamellae, the transverse processes, and the vertebral segments of the ribs on the other side [8]. The shape of this groove varies on the convex and concave sides of the curve, hence, the muscle system on both sides is different. Due to dislocations on the concave side resulting from spinous processes and the deviation towards the back because of lateral processes, the longest erector spinae muscle is displaced and is most often emphasized in the form of convex protrusion, sometimes up to the concave side. The iliocostalis muscle is associated with the paraspinal rib sections. The ribs in the rear hump are set in a plane similar to the sagittal one. The iliocostalis muscle at the top of the curve undergoes sagittal rotation in the course of the curvature, because above and below the hump, it lies in the frontal plane. The lateral fibers of this muscle attach to the ribs at the points of their angular bend in the area of the hump. The shift of the longest erector spinae muscle to the concave side and the rotation of the hippocampal muscle at the top of the curve causes a narrowing of their mass. On the concave side of the curve, the course of the erector spinae muscles is also changed. These muscles are arranged along the string of the curvature, and therefore, the longest back muscle, and especially the iliocostalis muscle on the concave side, are flattened and widened. In severe curvatures of the spine, macrophobia atrophy of the muscle fibers on the concave side and overgrowth with adipose tissue can be found [9].

In the lumbar section, there is a similar pattern of muscles on the convex and concave sides of the curve. The short erector spinae muscles forming the transversospinal system show smaller macro-lesions than the long muscles. The space between the transverse processes and spinous processes along the entire course of the right and left spine, despite its distortion, does not change and remains the same. This is related to the preservation of the original distance between the peaks of spinous and transverse processes, two or three lower vertebrae. The muscles of the transversospinal system, both on the convex and concave sides, change the direction of the fibers, but do not change their length. During surgical procedures in third degree scoliosis, degenerative changes of the transversospinal muscle system, pronounced overgrowth of adipose tissue are often found on the concave side of the curve. On the convex side, muscle mass is sometimes reduced, but they maintain a vivid-red color and do not show excessive adipose tissue. Histopathological tests performed during deep erector spinae muscle surgical procedures show degenerative changes on both sides of the curve, but they do explain whether they were primary or secondary, associated with the asymmetry of scoliotic spinal loads [9].

Surface electromyography (SEMG) examinations are used in work on the etiology of scoliosis, in the observation of its course in connection with attempts to identify patients at risk of progression. Evening the unequal tension of the erector spinae on both sides of the spine with physiotherapy causes a reduction in curvature angle and inhibition of the development of scoliosis [10]. Electromyography is an experimental technique related to obtaining, recording, and analyzing myoelectric signals. Myoelectric signals arise as a result of physiological changes in the state of the cell membranes of muscle fibers. Electromyography (EMG) measurements can be performed using intramuscular or surface injection electromyography. The advantages of surface electromyography include the possibility of performing any functional movements by the examined person during the measurement, easy assessment of muscle activity, non-invasiveness, painlessness, the possibility of selective assessment of various muscle groups at the same time, and high sensitivity to changes in muscle activity.

The raw SEMG amplitude is in the range between $( \pm) 5000 \mathrm{uV}$ [11]. SEMG records are converted using standard amplitude parameters such as average, peak, minimum value, area, and inclination. Before performing calculations, the record is cleared due to its bipolar nature. The value of the SEMG peak is only relevant for averaged curves, because even in the case of cleaned records, it is characterized by too high variability. The average value of the amplitude in the selected time interval is the most important parameter, because it is less sensitive to differences in the duration of the analyzed 
intervals [12]. From a clinical and practical point of view, the analysis (and interpretation) of the quality of SEMG amplitudes is the most important and the most useful EMG method for analyzing patient data. This type of examination is used to plan therapy and to evaluate its effectiveness. Electromyographic examinations carried out in the work on the etiology of scoliosis are in the mainstream of contemporary concepts of idiopathic scoliosis [13]. In SEMG, attention is paid to recorded abnormalities within the paraspinal muscles in the form of asymmetry of SEMG activity between the concave side and convex scoliosis. Analysis of the SEMG amplitude of the erector spinae can also be used to effectively recognize proper, helpful actions in corrective actions related to the work of scoliosis muscle patterns [14].

The aim of the study was to analyze the relationship between the angle of spinal curvature and the SEMG amplitude of the erector spinae in young school-children.

\section{Material and Methods}

A total of 251 children took part in the study, including $113(45.02 \%)$ girls and 138 (54.98\%) boys. There were $130(51.79 \%)$ 7-year-old children, including $63(48.46 \%)$ girls and $67(51.54 \%)$ boys. There were 121 (48.21\%) 8-year-old children, including 50 (41.32\%) girls and $71(58.68 \%)$ boys. The selection of subjects was mixed. The research was carried out in 2017 at the Posturology Laboratory of the Faculty of Medicine and Health Sciences, Jan Kochanowski University (UJK) in Kielce. All research procedures were carried out in accordance with the 1964 Declaration of Helsinki and with the consent of the University Bioethics Commission of the Jan Kochanowski University in Kielce (Resolution No. 5/2015). Body posture and the spine were examined using the optoelectronic method-Diers formetric III 4D—with raster stereography. Three-dimensional analysis of the spine is a combination of the newest techniques of optical imaging and digital data processing. This is a quick and non-contact $4 \mathrm{D}$ photogrammetry measurement and analysis of a patient's back and spine. The measurement results are very precise, and due to the fast image transfer to the computer, the data analysis takes place immediately after the test. Thanks to the measurements, a precise diagnosis is possible, enabling the selection of the best possible individual therapy.

The room in which the measurements were performed was darkened in a way that prevents direct sunlight falling on the patient's body [15]. At a distance of about $3 \mathrm{~m}$ from the optical tripod, a dark background was mounted. During the measurement, the patient was undressed to shorts and placed his/her back to the apparatus at a distance of $2 \mathrm{~m}$. The subject assumed habitual posture and the feet were placed in front of a line attached to the floor. The projector emitted horizontal stripes approximately $1 \mathrm{~cm}$ wide onto the patient's back. According to the Diers formetric III $4 \mathrm{D}$ manufacturer's recommendations, body posture examination was carried out with the DiCAM programme using the 'Average' measurement option. It consisted of taking a sequence of 12 film frames, which by creating an average value, reduced the variance of the posture and, thus, improving clinical value of the examination. The computer programme analyzed the data and determined the digital, photogrammetric image of body posture. Body posture examination was performed twice. The researcher (a specialist in postural re-education) decided which examination was more reflective of habitual posture, and only this test was further analyzed. Body posture examination with the Diers formetric III 4D device took about $15 \mathrm{~min}$. However, it was preceded by clinical examination, the Adams test was performed, and the length of the lower limbs was measured. According to the Diers formetric III 4D manufacturer guidelines, the presence of scoliosis and scoliotic posture was determined considering the values of three variables: pelvic tilt expressed in millimetres, lateral deviation expressed in millimetres, and surface rotation expressed in degrees.

Scoliotic posture occurred when pelvic tilt was $1-5 \mathrm{~mm}$, and at the same time, lateral deviation was 1-5 mm, while surface rotation was $1^{\circ}-5^{\circ}$. Scoliosis occurred when pelvic tilt and lateral deviation were greater than $5 \mathrm{~mm}(>5 \mathrm{~mm})$, while surface rotation was greater than 5 degrees $\left(>5^{\circ}\right)$ [16]. In the case that three requirements were not met, it was assumed that scoliosis or scoliotic posture did not occur.

After examining body posture, the children went to another room, where under the supervision of the supervisors, they rested for about $1 \mathrm{~h}$. Then, the SEMG amplitude test of the erector spinae 
was performed, which lasted about $30 \mathrm{~min}$. This analysis was performed using the Noraxon TeleMyo DTS 12 channel camera (Noraxon U.S.A., Inc., Scottsdale, AZ, USA). The device was certified by the EC (Certificate Production Quality Assurance Directive 93/42/EEC Medical Devices Annex V). This apparatus complies with the requirements of the 93/42/EEC Medical Devices Directive for class I products. It has the appropriate $\mathrm{CE}$ mark and corresponds to the definition of Class I devices and a product of the standard. Safety: IEC 60601-1 (1988) and EMC: IEC 60601-1-2 [17]. Pre-gel electrodes with a 3-cm diameter were used for the tests. The skin of the subjects was cleaned with abrasive liquid at the points where the electrodes were applied, so as to obtain resistance between the skin and the electrode below $2 \mathrm{k} \Omega$ [17]. The electrodes were located parallel to the direction of the tested muscle fibers. The distance between them was about 2 centimeters. The erector spinae muscle was examined in the thoracic and lumbar segments, both on the left and right side. Each of the trials lasted $10 \mathrm{~s}$.

After connecting the Noraxon device to the computer and the electrodes to the erector spinae muscle, each of the tests was preceded by entering the data for the examined person. Then, via the Noraxon TeleMyo DTS programme, the erector spinae was selected for analysis. Furthermore, a raw signal lasting about $15 \mathrm{~s}$ appeared on the computer screen, preceding the target recording of the muscle potential. Then, the measurement of the raw signal was recorded and analyzed. The researcher chose a signal modification programme consisting of cleaning the raw record from extreme, maximum, and minimum deflections. The examiner chose the option of processing the raw signal in order to calculate the average SEMG amplitude. Following, the processed mean amplitude of the SEMG of the extensor muscle of the spine in millivolts $(\mathrm{mV})$ was recorded. The results of the test took the scale of the voltage intensity in the time interval into account, which was $100 \mathrm{~ms}$. The study used the continuous trace recording mode. Functional potentials were recorded from the erector spinae in the thoracic and lumbar sections at the top of the curvature arches:

1. in habitual standing position (Frankfurt plane),

2. in resting position: in supine position (lower limbs straightened in the knee joints, upper limbs placed along the trunk),

3. under isometric contraction conditions:

- $\quad$ in supine position (lower limbs straightened in the knee joints, upper limbs placed along the trunk, pelvis stabilized), the subject lifts the trunk within the limits of the mobility of the spine, and then maintains this position for $10 \mathrm{~s}$,

- $\quad$ in supine position, with the upper trunk stabilized (shoulders and chest, limbs positioned as before), the subject lifts both lower limbs to the maximum possible height and maintains this position for $10 \mathrm{~s}$.

The SEMG amplitudes of the erector spinae were analyzed. This variable is used to assess the degree of activity and muscle tone. SEMG measurements were in line with SENIAM (Surface ElectroMyoGraphy for the Non-Invasive Assessment of Muscles) recommendation, a European research programme containing a number of guidelines regarding the selection of electrode types, their location, anatomy and muscle function, muscle group tests, signal processing, and hardware conditions [17]. Both the examination of body posture and spine as well as SEMG amplitude analysis were painless and non-invasive.

In order to determine the normality of the distribution of variables, the Kolmogorov-Smirnov test was carried out. In order to assess the differences in the size of the curvature of the spine, between girls and boys in scoliosis, scoliotic posture and the norm groups, SEMG amplitudes of the erector spinae in different study positions, one-way Analysis of Variance (ANOVA) was used. To determine the dependence and predictors between dependent variables, which were the types of postures and scoliosis angle, and the independent variable, that is the SEMG amplitude of the erector spinae, multiple stepwise and progressive regression models were used. Prior to regression analysis, k-fold cross validation was performed. The verification parameter used to assess the models was the coefficient 
of determination, the corrected coefficient of determination and the test statistic as well as the level of statistical significance, which clearly allowed to select models with the assumed level of statistical significance equaling $p<0.05$.

\section{Results}

In the study group, there were 103 (41\%) children with scoliosis, 141 (56.17\%) with scoliotic posture, and seven (3.0\%) with normal posture. The values of the position and dispersion measures for the curves had variable distributions in girls and boys in the scoliotic, scoliotic posture, and norm groups (Table 1). The greatest absolute differences in the angle of curvature occurred in the scoliosis group, both in girls $(S=10.72)$ and boys $(S=8.42)$. One-way analysis of variance showed significant differences between groups in the angle of curvature both in girls $(\mathrm{F}=46.6, p=0.001)$ and boys $(\mathrm{F}=61.81, p=0.001)$. This means that the values of the curves were significantly different between the scoliosis, scoliotic posture, and norm groups (Table 1).

Table 1. Differences in one-way Analysis of Variance (ANOVA) among scoliosis angle in girls and boys.

\begin{tabular}{|c|c|c|c|c|c|c|c|}
\hline \multicolumn{8}{|c|}{ Scoliosis Angle in Girls } \\
\hline \multirow{2}{*}{ Variables } & \multirow{2}{*}{ Mean } & \multicolumn{2}{|c|}{ Confidence } & \multirow{2}{*}{ Min } & \multirow{2}{*}{ Max } & \multirow{2}{*}{ SD } & \multirow{2}{*}{ ANOVA } \\
\hline & & $-95.00 \%$ & $95.00 \%$ & & & & \\
\hline Scoliosis & 22.64 & 19.17 & 26.12 & 9.00 & 51.00 & 10.72 & \multirow{3}{*}{$\begin{array}{l}\mathrm{F}=46.6 \\
p=\mathbf{0 . 0 0 1}\end{array}$} \\
\hline Scoliotic posture & 10.27 & 9.65 & 10.90 & 4.00 & 18.00 & 2.62 & \\
\hline Normal posture & 6.25 & 4.25 & 8.25 & 5.00 & 8.00 & 1.26 & \\
\hline \multicolumn{8}{|c|}{ Scoliosis Angle in Boys } \\
\hline \multirow{2}{*}{ Variables } & \multirow{2}{*}{ Mean } & \multicolumn{2}{|c|}{ Confidence } & \multirow{2}{*}{ Min } & \multirow{2}{*}{ Max } & \multirow{2}{*}{ SD } & \multirow{2}{*}{ ANOVA } \\
\hline & & $-95.00 \%$ & $95.00 \%$ & & & & \\
\hline Scoliosis & 20.59 & 18.49 & 22.70 & 11.00 & 47.00 & 8.42 & \multirow{3}{*}{$\begin{array}{l}\mathrm{F}=61.81 \\
p=\mathbf{0 . 0 0 1}\end{array}$} \\
\hline Scoliotic posture & 9.27 & 8.66 & 9.88 & 2.00 & 15.00 & 2.57 & \\
\hline Normal posture & 6.33 & 4.90 & 7.77 & 6.00 & 7.00 & 0.58 & \\
\hline
\end{tabular}

The values of the position and dispersion measures for SEMG amplitude of the erector spinae had diverse distributions in both girls and boys in the scoliosis, scoliotic posture, and normal posture groups. Among girls, the greatest absolute differentiation occurred for the variables supine position, lumbar section, right side in the scoliosis $(S=47.58)$, scoliotic posture $(S=47.22)$, and normal posture groups $(S=56.48)$. Among boys, the greatest absolute differentiation also occurred in the case of the variables supine position, lumbar section, right side, both in the scoliosis $(S=51.33)$ and scoliotic posture groups $(\mathrm{S}=47.00)$ (Table 2$)$.

Whereas in the group with the norm among boys, the highest absolute differentiation of values for the trunk variable was observed in the chest segment, right side $(S=48.29)$ (Table 3). In girls, the highest generalized amplitude was present in scoliosis $(x=47.175 \mathrm{mV})$, and also in boys with scoliosis $(x=48.726 \mathrm{mV})$.

One-way analysis of variance showed significant differences between groups in the size of SEMG amplitude of the erector spinae only in the case of boys. This concerned the amplitude in the test lower limbs up, lumbar section, left side ( $p=0.03)$. The values of SEMG amplitude distribution in this test differed significantly between the scoliosis, scoliotic posture and norm groups, and the significance level was less than $0.5(p<0.05)$ (Table 4$)$. 
Table 2. Surface electromyography (SEMG) amplitude of the erector spinae in girls.

\begin{tabular}{|c|c|c|c|c|c|c|}
\hline \multirow{2}{*}{ Variables } & \multicolumn{2}{|c|}{ Scoliosis } & \multicolumn{2}{|c|}{ Scoliotic Posture } & \multicolumn{2}{|c|}{ Correct Posture } \\
\hline & Mean mV & SD & Mean mV & SD & Mean mV & SD \\
\hline Standing position, chest segment, left side & 21.25 & 5.75 & 20.99 & 5.43 & 27.18 & 3.29 \\
\hline Standing position, chest segment, right side & 32.05 & 24.39 & 33.62 & 24.31 & 37.75 & 18.52 \\
\hline Standing position, lumbar section, left side & 20.73 & 9.85 & 22.40 & 10.10 & 19.29 & 10.34 \\
\hline Standing position, lumbar section, right side & 50.47 & 42.97 & 50.95 & 45.04 & 42.78 & 45.74 \\
\hline Supine position, chest segment, left side & 31.54 & 19.61 & 27.48 & 15.71 & 20.20 & 5.99 \\
\hline Supine position, chest segment, right side & 38.46 & 24.82 & 37.37 & 24.97 & 27.88 & 31.65 \\
\hline Lying on the left side, lumbar section & 20.96 & 14.38 & 19.73 & 13.53 & 11.56 & 14.57 \\
\hline Lying on the right side, lumbar section & 46.83 & 47.58 & 46.67 & 47.22 & 36.28 & 56.48 \\
\hline Torso up, chest segment, left side & 53.11 & 27.59 & 51.24 & 26.34 & 44.68 & 20.94 \\
\hline Torso up, chest segment, right side & 68.83 & 22.61 & 65.70 & 21.12 & 58.90 & 15.32 \\
\hline Torso up, lumbar section, left side & 52.53 & 27.07 & 48.36 & 22.52 & 55.23 & 20.99 \\
\hline Torso up, lumbar section, right side & 76.65 & 41.29 & 73.24 & 34.78 & 73.33 & 29.84 \\
\hline Lower limbs up, chest segment, left side & 36.42 & 18.68 & 34.08 & 17.07 & 32.03 & 13.96 \\
\hline Lower limbs up, chest segment, right side & 47.05 & 18.45 & 49.07 & 25.24 & 43.93 & 18.91 \\
\hline Lower limbs up, lumbar section, left side & 63.44 & 34.21 & 63.50 & 37.75 & 66.05 & 30.82 \\
\hline Lower limbs up, lumbar section, right side & 94.48 & 47.20 & 85.32 & 36.30 & 99.28 & 41.35 \\
\hline
\end{tabular}

Table 3. SEMG amplitude of the erector spinae in boys.

\begin{tabular}{|c|c|c|c|c|c|c|}
\hline \multirow{2}{*}{ Variables } & \multicolumn{2}{|c|}{ Scoliosis } & \multicolumn{2}{|c|}{ Scoliotic Posture } & \multicolumn{2}{|c|}{ Correct Posture } \\
\hline & Mean mV & SD & Mean mV & SD & Mean mV & SD \\
\hline Standing position, chest segment, left side & 23.61 & 12.72 & 19.97 & 8.35 & 14.72 & 4.22 \\
\hline Standing position, chest segment, right side & 32.35 & 24.42 & 30.48 & 25.81 & 11.51 & 3.88 \\
\hline Standing position, lumbar section, left side & 27.98 & 17.08 & 26.58 & 13.99 & 29.07 & 13.01 \\
\hline Standing position, lumbar section, right side & 53.63 & 48.23 & 49.98 & 44.03 & 31.77 & 3.16 \\
\hline Supine position, chest segment, left side & 24.75 & 11.55 & 22.75 & 10.20 & 22.43 & 8.40 \\
\hline Supine position, chest segment, right side & 37.71 & 26.31 & 31.58 & 23.85 & 18.53 & 6.90 \\
\hline Lying on the left side, lumbar section & 22.39 & 21.51 & 17.72 & 16.82 & 6.49 & 0.74 \\
\hline Lying on the right side, lumbar section & 47.84 & 51.33 & 41.92 & 47.00 & 8.60 & 1.53 \\
\hline Torso up, chest segment, left side & 56.01 & 31.07 & 53.73 & 25.91 & 75.80 & 47.76 \\
\hline Torso up, chest segment, right side & 67.76 & 29.11 & 69.65 & 23.73 & 76.23 & 48.29 \\
\hline Torso up, lumbar section, left side & 55.36 & 28.82 & 51.89 & 24.76 & 61.77 & 15.50 \\
\hline Torso up, lumbar section, right side & 78.55 & 39.26 & 74.08 & 33.65 & 63.97 & 12.67 \\
\hline Lower limbs up, chest segment, left side & 38.60 & 23.08 & 40.55 & 24.20 & 46.50 & 26.92 \\
\hline Lower limbs up, chest segment, right side & 53.61 & 34.41 & 56.05 & 33.04 & 40.60 & 26.50 \\
\hline Lower limbs up, lumbar section, left side & 64.47 & 34.76 & 62.73 & 30.46 & 114.23 & 28.46 \\
\hline Lower limbs up, lumbar section, right side & 95.00 & 42.18 & 91.86 & 32.39 & 100.97 & 2.05 \\
\hline
\end{tabular}

Table 4. Analysis of ANOVA differences for SEMG amplitude of the erector spinae between the scoliotic, scoliotic posture, and normal posture groups of boys and girls.

\begin{tabular}{ccccc}
\hline Variables & \multicolumn{2}{c}{ Girls } & \multicolumn{2}{c}{ Boys } \\
\cline { 2 - 5 } & $\mathbf{F}$ & $\boldsymbol{p}$ & $\mathbf{F}$ & $\boldsymbol{p}$ \\
\hline Standing position, chest segment, left side & 2.40 & 0.10 & 2.64 & 0.08 \\
Standing position, segment right, side & 0.13 & 0.88 & 1.02 & 0.36 \\
Standing position, lumbar section, left side & 0.47 & 0.63 & 0.16 & 0.85 \\
Standing position, lumbar section, right side & 0.06 & 0.94 & 0.39 & 0.68 \\
Supine position, chest segment, left side & 1.22 & 0.30 & 0.59 & 0.55 \\
Supine position, chest segment, right side & 0.32 & 0.73 & 1.63 & 0.20 \\
Lying on the left side, lumbar section & 0.84 & 0.43 & 1.74 & 0.18 \\
Lying on the right side, lumbar section & 0.09 & 0.91 & 1.05 & 0.35 \\
Torso up, chest segment, left side & 0.20 & 0.82 & 0.88 & 0.42 \\
Torso up, chest segment, right side & 0.53 & 0.59 & 0.20 & 0.82 \\
Torso up, lumbar section, left side & 0.47 & 0.63 & 0.43 & 0.65 \\
Torso up, lumbar section, right side & 0.11 & 0.90 & 0.42 & 0.65 \\
Lower limbs up, chest segment, left side & 0.27 & 0.76 & 0.24 & 0.79 \\
Lower limbs up, chest segment, right side & 0.17 & 0.85 & 0.35 & 0.70 \\
Lower limbs up, lumbar section, left side & 0.01 & 0.99 & 3.61 & $\mathbf{0 . 0 3}$ \\
Lower limbs up, lumbar section, right side & 0.77 & 0.46 & 0.18 & 0.83 \\
\hline
\end{tabular}




\subsection{Relationship between Body Posture and SEMG Amplitude of the Erector Spinae}

The independent variables were SEMG amplitude of the erector spinae, while the dependent variables were the posture of the body and spine. The regression model for the group with the norm and the group with scoliotic posture did not reach the assumed statistical significance level, therefore, it was not shown and or considered correct in modelling the relationship between body postural variables and SEMG amplitude of the erector spinae in various positions. The most important and statistically significant predictors for the body posture variables and SEMG amplitude of the erector spinae examined in various positions in the scoliosis group were the variables lower limbs up, lumbar section, left side ( $p=0.01)$; lower limbs up, lumbar section, right side $(p=0.05)$; standing position, chest segment, left side ( $p=0.01)$; and standing position, chest segment, right side $(p=0.03)$. The variance explained by the independent variables adopted in the model accounted for $43 \%$ of the total volatility $\left(R^{2}=0.43\right)$, which indicates average adjustment to the data, however, the assumed level of statistical significance $(p=0.01)$ and the appropriate value of the statistical test were obtained $\mathrm{F}=2.94$ (Table 5 ).

Table 5. Linear stepwise regression model for SEMG amplitude of the erector spinae in children with scoliosis ${ }^{1}$.

\begin{tabular}{|c|c|c|c|c|c|c|}
\hline \multicolumn{7}{|c|}{ Linear Stepwise Regression Model for SEMG Amplitude of the Erector Spinae and Scoliosis } \\
\hline Variable & $\mathbf{b}^{*}$ & Std. Error $\mathbf{Z} \mathbf{b}^{*}$ & b & Std. Error Z b & $t(95)$ & $p$ \\
\hline Constant & & & 1.09 & 0.68 & 1.59 & 0.11 \\
\hline Lower limbs up, lumbar section, left side & 0.36 & 0.13 & 0.01 & 0.00 & 2.77 & 0.01 \\
\hline Lower limbs up, lumbar section, right side & -0.29 & 0.15 & -0.01 & 0.01 & -2.00 & 0.05 \\
\hline Standing position, chest segment, left side & 0.56 & 0.21 & 0.04 & 0.01 & 2.71 & 0.01 \\
\hline Standing position, chest segment, right side & -0.42 & 0.19 & -0.03 & 0.01 & -2.18 & 0.03 \\
\hline \multicolumn{7}{|c|}{$\mathrm{R}=0.43 \mathrm{R}^{2}=0.18 ;$ Correction $\mathrm{R}^{2}=0.12 ; \mathrm{F}(7.91)=2.94 ; p=\mathbf{0 . 0 1}$} \\
\hline \multicolumn{7}{|c|}{$\begin{array}{l}\text { Linear Stepwise Regression Model for SEMG Amplitude of the Erector Spinae and Curvature Angle in the } \\
\text { Scoliosis Group }\end{array}$} \\
\hline Variable & $\mathbf{b}^{*}$ & Std. Error $\mathbf{Z} \mathbf{b}^{*}$ & $\mathbf{b}$ & Std. Error Z b & $t(95)$ & $p$ \\
\hline Constant & & & 10.14 & 0.90 & 11.27 & 0.001 \\
\hline Supine position, lumbar section, left side & 0.25 & 0.09 & 0.03 & 0.01 & 2.69 & 0.01 \\
\hline
\end{tabular}

$$
\mathrm{R}=0.24 ; \mathrm{R}^{2}=0.06 \text { Correction } \mathrm{R}^{2}=0.04 ; \mathrm{F}(3.137)=2.9 ; p=\mathbf{0 . 0 4}
$$

${ }^{1} \mathrm{~b}^{*}$-constant regression, Std. Error $\mathrm{Z} \mathrm{b}^{*}$ - standard error of the regression constant; $\mathrm{b}$ - the partial regression coefficients, Std. Error Z b-standard error of the partial regression coefficient, $t$-statistical test, $p$-level of significance.

\subsection{Angle of Spinal Curvature and Frequency of Erector Spinae SEMG}

The SEMG amplitude of the erector spinae was an independent variable, while the dependent variable was the value of the angle of spinal curvature. The regression model for the group with the norm and scoliotic posture did not reach the assumed statistical significance level, therefore, it was not shown or considered correct in the modelling of the relationship between SEMG amplitude of the erector spinae and the angle of curvature. The most important and statistically significant predictor of SEMG amplitude and the angle of curvature in the scoliosis group was standing position, chest segment, right side $(p=0.01)$. The variance explained by the independent variables adopted in the model accounted for $32 \%$ of the total volatility $\left(R^{2}=0.32\right)$, which indicated small adjustment to the data, however, the assumed statistical significance level $(p=0.01)$ was met and also, the appropriate value for the statistical test was obtained $\mathrm{F}=3.15$ (Table 5).

\section{Discussion}

The presented research indicates the validity of the concept of the formation and development of idiopathic scoliosis as a consequence of the asymmetry of erector spinae muscle tone. Since the beginning of research on erector spinae EMG examination, it has been noticed that there is a relationship between the size of the spinal curvature angle and the uneven tension of the erector spinae muscles on 
both sides of the spine. Therefore, the goal of scoliosis therapy should be to restore the proper tension of these muscles. Disturbances in the balance of muscle tension can trigger a set of changes that create a clinical and anatomopathological image of scoliosis.

In this study, it was observed that the largest generalized SEMG amplitude of the erector spinae muscles is present in scoliosis. The larger, asymmetrical activity of the erector spinae visible in the SEMG amplitude record is associated with an increase in the angle of the spinal curvature. The increase of SEMG amplitude on the side of curvature protrusion—both during rest and movement-was observed by many researchers [18]. However, the interpretation of this phenomenon occurs in most of the cases studied.

Bayer [19] believed that idiopathic scoliosis arises as a result of the asymmetrical operation of the erector spinae muscle, the bioelectric activity of which, greater on the convex side, is the cause of deformation. In the treatment of thoracic scoliosis, the author carried out de-innervation of the erector spinae muscle by resection of the posterior spinal nerve from Th7 to Th10 on the side of curvature.

Roaf [20-22] claimed that the cause of idiopathic scoliosis is the imbalance of muscles rotating the spine on the side of the protruding curvature.

Henssge [23], Redford et al. [24] and Żuk [25] interpret the phenomenon of excessive bioelectrical muscle activity of the convex side as exaggerated reflex to stretching, as a compensation mechanism, or fatigue reaction. Increased bioelectrical activity on the side of the protruding curvature is a synonym of progressivity for some researchers, because in compensated scoliosis and after spondylodesis, bioelectric activity disappears.

Meanwhile, Güth and Abbink [26] found differences of bioelectrical activity in the paravertebral muscles in the case of non-aggressive curvatures during static and dynamic loading of the upper limbs. When performing maximal isometric work, the differences in bioelectrical activity between the convex and concave sides are smaller, which suggests that in idiopathic scoliosis, the etiological agent must not be muscle weakness on the side of the concave curvature.

Filder et al. [27] concluded that the cause of idiopathic scoliosis is excessive activity of the deep erector spinae muscles on the side of the convex curvature. The activity of the back muscles during constant loading is greater in children with scoliosis than in healthy subjects. In both cases, the bioelectrical activity of the muscles in the thoracic segment was higher than in the lumbar region and the asymmetry of the tension was stronger in the chest segment on the right side [28,29].

Polster [30] presented another approach to neuromuscular imbalance as a cause of scoliosis. This author emphasizes the role of neuromuscular functional unity in the process of blastomere formation. Congenital disorders in the differentiation of nerve structures or myotomes can cause muscular-nerve imbalance on both sides of the spine, resulting in its segmental structural changes. According to some researchers, dysfunction of the peripheral neuron underlies idiopathic scoliosis.

Hennssge [23] found the occurrence of fibrillation, especially in curves below $20^{\circ} \mathrm{Cobb}$ angle and multiphase potentials in $30 \%$ of subjects on both the concave and convex sides.

Kaplan et al. [31], and Żuk and Tokarowsk [32] describe fibrillation on the side of curvature convexity. Siato [33] reports that in idiopathic scoliosis, where the pathological potentials in the form of fibrillation were electromyographically observed, degenerative changes were found in the erector spinae muscle junctions of on the side of curvature convexity.

Other authors, including Aleksander et al. [34], Güth and Abbink [26], and Weiss et al. [35], did not notice idiopathic resting activity in the form of fibrillation, fasciculation, or positive waves in scoliosis. In addition, Güth and Abbink [26] believe that these phenomena are not evidence of de-innervation unless they are found in many places in the muscle under examination.

In another study, the effect of a several-week long rehabilitation programme using the Schroth method on erector spinae tone in children with scoliosis was evaluated. Significant reductions in muscle activity by $6.85 \%$ in the thoracic convex region and totaling $14.2 \%$ on the lumbar convex side were found. The activity quotient (convex/concave) was reduced by $11.99 \%$ in the thoracic region and by $7.91 \%$ in the lumbar region. These findings confirm the improvement of postural performance capacity 
after the intensive in-patient Schroth rehabilitation programme. As the imbalance of electromyographic activity may be influenced by scoliosis-specific exercises leading to a highly significant reduction of the Cobb angle, it is assumed to be secondary to the development of the scoliotic curve and may not be a primary factor in the etiology of idiopathic scoliosis [36]. On the other hand, in the given study, EMG responses of the erector spinae to postural perturbation have been described and interpreted as unloading reflex. Moreover, these responses have been found to be clearly and constantly abnormal in subjects presenting progressive idiopathic scoliosis when compared to responses observed in subjects presenting non-progressive scoliosis or in normal subjects. In order to investigate responses and obtain more precise information on their components, origin and variations in scoliotic children, the subjects stood on a specially constructed platform that could be suddenly tilt either to the right or to the left. Thoracic and lumbar paraspinal muscle activity was recorded with pairs of self-adhesive surface bipolar EMG electrodes. The responses were analyzed to detect components and study their time course and relative amplitude in successive trials; characteristics common to different subjects were sought. The presence of short-latency responses and later activities following postural perturbation was confirmed. In a given subject, these components vary in amplitude and time course from one trial to another. On the other hand, the differences found across subjects are not significantly different from those found within the various subjects. Results exhibit some differences with previous data. They lead to a different neurophysiological interpretation and indicate that the stimulus and the responses need more precise analysis before being used as a diagnostic and prognostic tool in evolutive scoliosis [37].

The aim of another study was to evaluate electromyographic (EMG) responses of erector spinae (ES) and lower limb muscles to dynamic forward postural perturbation (FPP) and backward postural perturbation (BPP) in patients with adolescent idiopathic scoliosis (AIS) and in a healthy control group. Muscle responses were measured over a 1s time window after the onset of perturbation. In the FPP test, the EMG responses of right EST10, ESL3, and biceps femoris (BF) muscles in the scoliosis group were respectively about 1.40,1.43, and 1.45 times greater than those in the control group. Additionally, in the BPP test, right ESL3 muscle of the scoliosis group, the EMG activity was 1.64 times higher than that in the control group. During FPP, the scoliosis group displayed asymmetrical muscle responses in the EST10 and BF muscles. This asymmetrical muscle activity in response to FPP is hypothesized to be a possible compensatory strategy rather than an inherent characteristic of scoliosis [38].

The uneven tension of the erector spinae in children with scoliosis, visible in SEMG amplitude, causes its destabilization and abnormal development.

\section{Conclusions}

Only in the group of boys were significant group-related differences found in SEMG amplitude of the erector spinae. This concerned SEMG amplitude in the test: lower limbs up, lumbar section, left side. The values of SEMG amplitude distribution in this test differed significantly between the scoliosis, scoliotic posture, and normal posture groups. The greatest generalized SEMG amplitude of the erector spinae occurred in scoliosis. The most important and statistically significant predictors of body posture and SEMG amplitude of the erector spinae examined in various positions in the scoliosis group turned out to be the variables lower limbs up, lumbar section left side; lower limbs up, lumbar section, right side; standing position, chest segment, left side; and standing position, chest segment, right side. A significant correlation was found between the angle of spinal curvature and the SEMG amplitude of the erector spinae. The most important and statistically significant predictor of SEMG amplitude and the angle of scoliosis in the scoliosis group was the standing position, chest segment, right side. The largest generalized SEMG amplitude of the erector spinae occurred in both boys and girls with scoliosis. Disturbing the balance of muscle tension in the erector spinae can trigger a set of changes that create a clinical and anatomopathological image of the curvature of the spine.

Author Contributions: J.W.: Conceptualization, Data curation, Formal analysis, Methodology, Writing-original draft; P.K.: Data curation, Formal analysis; S.J.: Data curation; M.K.: Data curation; N.H.-T.: Writing-review and editing. 
Funding: The project is supported under the program of the Minister of Science and Higher Education under the name "Regional Initiative of Excellence" in 2019-2022 project number: 024/RID/2018/19, financing amount: 11.999.000 PLN.

Conflicts of Interest: The authors declare no conflict of interest regarding the publication of this paper.

\section{References}

1. Kotwicki, T.; Chowanska, J.; Kinel, E.; Czaprowski, D.; Tomaszewski, M.; Janusz, P. Optimal management of idiopathic scoliosis in adolescence. Adolesc. Health Med. Ther. 2013, 4, 59-73. [CrossRef] [PubMed]

2. Głowacki, M.; Kotwicki, T.; Pucher, A. Skrzywienie kręgosłupa. In Wiktora Degi Ortopedia i Rehabilitacja; Red, W., Marciniak, A., Szulc, Eds.; PZWL: Warszawa, Poland, 2008.

3. Archer, I.A.; Dickson, R.A. Stature and idiopathic scoliosis a prospective study. J. Bone Jt. Surg. 1985, 67, 185-188. [CrossRef]

4. Alici, E.; Pinar, H. The Alici spinal system in the surgical treatment of scoliosis. Acta Orthop. Belg. 1992, 58, 122-128. [PubMed]

5. Dubousset, J.; Chopin, D.; Seringe, R. Have we made true progress in surgical indications and determining the limitations of spinal fusion in patients with idiopathic scoliosis? Orthop. Traumatol. Surg. Res. 2018, 104, 555-556. [CrossRef] [PubMed]

6. Kobayashi, K.; Imagama, S.; Ito, Z.; Ando, K.; Hida, T.; Ito, K.; Tsushima, M.; Ishikawa, Y.; Matsumoto, A.; Nishida, Y.; et al. Transcranial motor evoked potential waveform changes in corrective fusion for adolescent idiopathic scoliosis. J. Neurosurg. Pediatr. 2017, 19, 108-115. [CrossRef] [PubMed]

7. Kwok, G.; Yip, J.; Cheung, M.C.; Yick, K.L. Evaluation of Myoelectric Activity of Paraspinal Muscles in Adolescents with Idiopathic Scoliosis during Habitual Standing and Sitting. Biomed. Res. Int. 2015, 2015, 958450. [CrossRef] [PubMed]

8. Ko, J.Y.; Suh, J.H.; Kim, H.; Ryu, J.S. Proposal of a new exercise protocol for idiopathic scoliosis: A preliminary study. Medicine (Baltimore) 2018, 97, e13336. [CrossRef] [PubMed]

9. Tylman, D. Pathomechanics of Lateral Spinal Curvatures; Severus: Warsaw, Poland, 1995.

10. Zetterberg, C.; Bjüdrk, R.; Örtengren, R.; Andersson, G.B.J. Elektromyography of the paravertebral muscles in idiopathic scoliosis. Acta Orthop. Scand. 1984, 55, 304. [CrossRef] [PubMed]

11. Valentino, B.; Maccauro, L.; Mango, G.; Melito, F.; Fabozzo, A. Electromyography for the investigation and early diagnosis of scoliosis. Anat Clin. 1985, 7, 55-59. [CrossRef]

12. Stetkarova, I.; Zamecnik, J.; Bocek, V.; Vasko, P.; Brabec, K.; Krbec, M. Electrophysiological and histological changes of paraspinal muscles in adolescent idiopathic scoliosis. Eur. Spine J. 2016, 25, 3146-3153. [CrossRef]

13. Feipel, V.; Aubin, C.E.; Ciolofan, O.C.; Beauséjour, M.; Labelle, H.; Mathieu, P.A. Electromyogram and kinematic analysis of lateral bending in idiopathic scoliosis patients. Med. Biol. Eng. Comput. 2002, 40, 497-505. [CrossRef] [PubMed]

14. Guo, L.Y.; Wang, Y.L.; Huang, Y.H.; Yang, C.H.; Hou, Y.Y.; Harn, H.I.; You, Y.L. Comparison of the electromyographic activation level and unilateral selectivity of erector spinae during different selected movements. Int. J. Rehabil. Res. 2012, 35, 345-351. [CrossRef] [PubMed]

15. Hierholzer, E.; Drerup, B. Influence of length discrepancy on ratserstereographic back shape parameters. Orthopade 2001, 30, 242-250.

16. Humbert, L.; Steffen, J.S.; Vialle, R.; Dubousset, J.; Vital, J.M.; Skalli, W. 3D analysis of congenital scoliosis due to hemivertebra using biplanar radiography. Eur. Spine J. 2013, 22, 379-386. [CrossRef] [PubMed]

17. Wilczyński, J.; Karolak, P.; Janecka, S. Body composition and SEMG amplitude of the spine rectifier in children with scoliotic lesions. J. Educ. Health Sport 2018, 8, 600-612. [CrossRef]

18. Dobosiewicz, K. Neurophysiological mechanism of the unloading reflex as a prognostic factor in the early stages of idiopathic adolescent scoliosis. Eur. Spine J. 1997, 6, 93-97. [CrossRef] [PubMed]

19. Bayer, H. Das Verhalten der Rückenstrecker bei einem Fall von konvexseitiger Denervierungsoperation bei Skoliose. Z. Orthop. 1980, 118, 274. [CrossRef] [PubMed]

20. Roaf, R. Rotation movements of the spine with special reference to scoliosis. J. Bone Jt. Surg. 1958, 408, 312-332. [CrossRef]

21. Roaf, R. The postural function of intercostal muscle. Zorab. PA, 1974; 46.

22. Roaf, R. The basic anatomy of scoliosis. J. Bone Jt. Surg. Br. 1966, 488, 786-792. [CrossRef] 
23. Henssge, J. Electromyographic contribution to the problem of scoliosis. Z. Orthop. Ihre Grenzgeb. 1964, 99, 167-195.

24. Redford, J.B.; Butterworth, T.R.; Clements, E.L., Jr. Use of electromyography as a prognostic aid in the management of idiopathic scoliosis. Arch. Phys. Med. Rehabil. 1969, 50, 433-438. [PubMed]

25. Żuk, T. Etiology and pathogenesis of idiopathic scoliosis from the viewpoint of electromyographic studies. Beitr. Orthop. Traumatol. 1965, 12, 138-141. [PubMed]

26. Güth, V.; Abbink, F. Electromyographical and kinesiological investigations: Comparison of congenital and idiopathic scoliosis. Z. Orthop. Ihre Grenzgeb. 1980, 118, 165-172. [CrossRef] [PubMed]

27. Fidler, M.W.; Jowett, R.L. Muscle Imbalance in the Aetiology of Scoliosis. J. Bone Jt. Surg. Br. 1976, 58-B, 20020. [CrossRef]

28. Hoogmartens, M.J.; Basmajian, J.V. Asymmetrical postural tone as a possible cause of idiopathic scoliosis. A preliminary communcation. Electromyogr. Clin. Neurophysiol. 1973, 13, 365-366. [PubMed]

29. Reuber, M.; Schultz, A.; McNeill, T.; Spencer, D. Trunk muscle myoelectric activities in idiopathic scoliosis. Spine 1983, 8, 447-456. [CrossRef] [PubMed]

30. Polster, J. New data on the correction of scoliosis. Z. Orthop. Ihre Grenzgeb. 1976, 114, 447-452. [PubMed]

31. Kaplan, P.E.; Sahgal, V.; Hughes, R.; Kane, W.; Flanagan, N. Neuropathy in thoracic scoliosis. Acta Orthop. Scand. 1980, 51, 263-266. [CrossRef]

32. Żuk, T.; Tokarowski, A.; Pathomechanics Donisch, E.W.; Basmajian, J.V. Electromyography of deep back muscles in man. Am. J. Anat. 1972, 133, 25-36.

33. Sato, H. Functional characteristics of human skeletal muscle revealed by spectral analysis of the surface electromyogram. Electromyogr. Ciln. Neurophyslol. 1982, 22, 459.

34. Alexander, M.A.; Seoson, E.H. Idiopathic scoliosis: An electromygraphic study. Arch. Phys. Med. Rehabil. 1978, 59, 314-315. [PubMed]

35. Weiss, M.; Milkowska, A.; Kozinska, M. Conservative treatment of scoliosis in the light of electromyographic data. Chir. Narz. Ruchu Ortop. Polska 1957, 22, 197-209. [PubMed]

36. Weiss, H.R. Imbalance of electromyographic activity and physical rehabilitation of patients with idiopathic scoliosis. Eur. Spine J. 1993, 1, 240-243. [CrossRef] [PubMed]

37. Perret, C.; Robert, J. Electromyographic responses of paraspinal muscles to postural disturbance with special reference to scoliotic children. J. Manip. Physiol. Ther. 2004, 27, 375-380. [CrossRef] [PubMed]

38. Farahpour, N.; Ghasemi, S.; Allard, P.; Saba, M.S. Electromyographic responses of erector spinae and lower limb's muscles to dynamic postural perturbations in patients with adolescent idiopathic scoliosis. J. Electromyogr. Kinesiol. 2014, 24, 645-651. [CrossRef] [PubMed] 